\title{
Quantum fluctuations of the Chern-Simons theory and dynamical dimensional reduction
}

\author{
Ivan Andrić, Velimir Bardek, and Larisa Jonke* \\ Theoretical Physics Division, \\ Rudjer Bošković Institute, P.O. Box 1016, \\ 10001 Zagreb, CROATIA
}

\begin{abstract}
We consider a large-N Chern-Simons theory for the attractive bosonic matter (Jackiw-Pi model) in the Hamiltonian collective-field approach based on the $1 / \mathrm{N}$ expansion. We show that the dynamics of low-lying density excitations around the ground-state vortex configuration is equivalent to that of the Sutherland model. The relationship between the Chern-Simons coupling constant $\lambda$ and the Calogero-Sutherland statistical parameter $\lambda_{s}$ signalizes some sort of statistical transmutation accompanying the dimensional reduction of the initial problem.
\end{abstract}

PACS number(s): 11.10.Lm 74.20.Kk 03.65.Sq 05.30.-d

Gauge models of a scalar field with the Chern-Simons term [1] in 2+1 space-time dimensions are known to support soliton or vortex solutions [2, [3]. By using the nonrelativistic field theory of the self-attracted bosonic matter minimally coupled to an Abelian Chern-Simons

\footnotetext{
*e-mail address: andric@thphys.irb.hr

bardek@thphys.irb.hr

larisa@thphys.irb.hr
} 
gauge field, the authors of Ref. [2] have shown that there exists a static self-dual soliton solution for a specific choice of the coupling constant. We have rederived this soliton solution in the collective-field approach by including higher-order terms in the $1 / N$ expansion $\mathbb{4}$. In our approach, this soliton solution saturates the Bogomol'nyi bound and does not receive quantum corrections to its energy in the next-to-leading approximation.

In this paper we analyze the quantum dynamics of low-lying density fluctuations around a specific vortex solution and show that it is equivalent to the dynamics of quantum fluctuations in the Calogero-Sutherland model [5]. There exist a number of papers [6] that elucidate the connection between the Chern-Simons-based anyonic physics in the fractional quantum Hall effect and the Calogero-Sutherland model, but it should be emphasized that we are working in a completely different physical situation. We are trying to establish a dynamical reduction of the Jackiw-Pi model to the Calogero-Sutherland one. In Ref. [7] we conjectured the form of quantum fluctuations in the Jackiw-Pi model and that allowed us to identify the dynamics of these fluctuations with those of the Calogero-Sutherland model. In this paper, we are looking for the same result using a different approach and thus indirectly confirming the conjecture made in [7].

We begin our analysis of the Jackiw-Pi model by repeating the main results of Ref. [4]. The collective-field approach to the model is described by the Hamiltonian

$$
H=\frac{1}{2} \int d^{2} \mathbf{r} \rho(\mathbf{r})\left[\nabla \pi(\mathbf{r})+\hat{n} \times\left(\frac{1}{2} \frac{\nabla \rho(\mathbf{r})}{\rho(\mathbf{r})}+|\lambda| \int d^{2} \mathbf{r}^{\prime} \rho\left(\mathbf{r}^{\prime}\right) \frac{\mathbf{r}-\mathbf{r}^{\prime}}{\left|\mathbf{r}-\mathbf{r}^{\prime}\right|^{2}}-v \frac{\mathbf{r}-\mathbf{R}}{|\mathbf{r}-\mathbf{R}|^{2}}\right)\right]^{2}
$$

where $\hat{n}$ is the unit vector perpendicular to the plane in which particles move, the dimensionless constant $\lambda$ is the so-called statistical parameter, the vorticity $v$ is a dimensionless integer, and $\pi(\mathbf{r})$ is the canonical conjugate of the collective field $\rho(\mathbf{r})$ :

$$
\left[\nabla \pi(\mathbf{r}), \rho\left(\mathbf{r}^{\prime}\right)\right]=-i \nabla \delta\left(\mathbf{r}-\mathbf{r}^{\prime}\right)
$$

The leading part of the collective-field Hamiltonian in the $1 / N$ expansion is given by the effective potential

$$
V_{\mathrm{eff}}=\frac{1}{2} \int d^{2} \mathbf{r} \rho(\mathbf{r})\left(\frac{1}{2} \frac{\nabla \rho(\mathbf{r})}{\rho(\mathbf{r})}+|\lambda| \int d^{2} \mathbf{r}^{\prime} \rho\left(\mathbf{r}^{\prime}\right) \frac{\mathbf{r}-\mathbf{r}^{\prime}}{\left|\mathbf{r}-\mathbf{r}^{\prime}\right|^{2}}-v \frac{\mathbf{r}-\mathbf{R}}{|\mathbf{r}-\mathbf{R}|^{2}}\right)^{2} .
$$


Owing to the positive definiteness of the effective potential (3), the Bogomol'nyi limit appears. The Bogomol'nyi bound is saturated by the positive normalizable solution $\rho_{0}(\mathbf{r})$ of the Liouville-type equation

$$
\Delta \ln \rho_{0}(\mathbf{r})+4|\lambda| \pi \rho_{0}(\mathbf{r})=4 \pi v \delta(\mathbf{r})
$$

It has been shown in [4] that there exists a radially symmetric, positive, and normalizable collective-field configuration that minimizes the energy (1). It is given by the vortex form

$$
\rho_{0}(r)=\frac{|\lambda| N^{2}}{2 \pi r^{2}}\left[\left(\frac{r_{0}}{r}\right)^{\frac{N|\lambda|}{2}}+\left(\frac{r}{r_{0}}\right)^{\frac{N|\lambda|}{2}}\right]^{-2} .
$$

The vorticity $v$ is fixed by the normalization condition and is given by

$$
v=N \frac{|\lambda|}{2}-1
$$

The parameter $r_{0}$ reflects the scale invariance of the problem and cannot be determined. Now, if $\mathrm{N}$ is large enough, we can replace the soliton configuration $\rho_{0}(r)$ by the $\delta$ profile:

$$
\rho_{0}(r)=\frac{N}{2 \pi} \frac{\delta\left(r-r_{0}\right)}{r_{0}}
$$

Here we have used the well-known representation of the $\delta$-function:

$$
\delta(x)=\lim _{\epsilon \rightarrow 0} \frac{\exp (x / \epsilon)}{\epsilon[1+\exp (x / \epsilon)]^{2}}, \epsilon=\frac{1}{N|\lambda|} .
$$

At this point we analyze the dynamics of the collective-field excitations around the groundstate solution of the Jackiw-Pi model. We perform the $1 / N$ expansion of the collective field $\rho(\mathbf{r})$ in the form

$$
\rho(\mathbf{r})=\rho_{0}(\mathbf{r})+\eta(\mathbf{r})
$$

where $\rho_{0}(\mathbf{r})$ is the ground-state semiclassical configuration and $\eta(\mathbf{r})$ a small density quantum fluctuation around $\rho_{0}(\mathbf{r})$. Inserting (9) in (11) and expanding in $\eta(\mathbf{r})$, we obtain the leading term $V_{\text {eff }}\left(\rho_{0}\right)$ and the Hamiltonian quadratic in fluctuations and its canonical conjugate. After introducing the operators 


$$
\begin{aligned}
A(z) & =\frac{\partial \pi}{\partial z}-i \frac{\partial}{\partial z}\left(\frac{\eta}{2 \rho_{0}}+|\lambda| \int d^{2} \mathbf{r}^{\prime} \ln \left|z-z^{\prime}\right| \eta\left(z^{\prime}\right)\right) \\
A^{\dagger}(z) & =\frac{\partial \pi}{\partial \bar{z}}+i \frac{\partial}{\partial \bar{z}}\left(\frac{\eta}{2 \rho_{0}}+|\lambda| \int d^{2} \mathbf{r}^{\prime} \ln \left|z-z^{\prime}\right| \eta\left(z^{\prime}\right)\right)
\end{aligned}
$$

with the c-number commutator (all other vanishing)

$$
\left[A(z), A^{\dagger}\left(z^{\prime}\right)\right]=2 \frac{\partial^{2}}{\partial z \partial \bar{z}^{\prime}}\left(\frac{\delta^{2}\left(z-z^{\prime}\right)}{2 \rho_{0}}+|\lambda| \ln \left|z-z^{\prime}\right|\right)
$$

we are left with a Hamiltonian that governs the dynamics of low-lying excitations in the form:

$$
H=2 \int d^{2} \mathbf{r} \rho_{0}(z) A^{\dagger}(z) A(z)
$$

There are some subtleties involving the ordering of the operators $A$ and $A^{\dagger}$, but an interested reader can find all details in Ref. [4].

Now, to find the spectrum of low-lying excitations, we have to diagonalize the Hamiltonian (12). We expand the operators $A$ and $A^{\dagger}$ in terms of a new, complete set of operators

$$
A(z)=\sum_{\mathbf{n}} \phi_{\mathbf{n}}(z) a_{\mathbf{n}}, A^{\dagger}(z)=\sum_{\mathbf{n}} \phi_{\mathbf{n}}^{*}(z) a_{\mathbf{n}}^{\dagger}
$$

that satisfy the standard bosonic commmutation relations

$$
\left[a_{\mathbf{n}}, a_{\mathbf{m}}^{\dagger}\right]=\delta_{\mathbf{n}, \mathbf{m}},\left[a_{\mathbf{n}}, a_{\mathbf{m}}\right]=\left[a_{\mathbf{n}}^{\dagger}, a_{\mathbf{m}}^{\dagger}\right]=0
$$

We demand that the Hamiltonian (12) should take the diagonal form

$$
H=\sum_{\mathbf{n}} \omega_{\mathbf{n}} a_{\mathbf{n}}^{\dagger} a_{\mathbf{n}}
$$

Here, $\mathbf{n}$ represents a pair of quantum numbers, and it is assumed that the sum is replaced by an integral in the nondiscrete case. We insert expansion (13) in the commutator (11) and apply (14) to obtain the completeness relation:

$$
\sum_{\mathbf{n}} \phi_{\mathbf{n}}(z) \phi_{\mathbf{n}}^{*}\left(z^{\prime}\right)=2 \frac{\partial^{2}}{\partial z \partial \bar{z}^{\prime}}\left(\frac{\delta^{2}\left(z-z^{\prime}\right)}{2 \rho_{0}}+|\lambda| \ln \left|z-z^{\prime}\right|\right) .
$$

Inserting (13) in (12), and demanding (15), we obtain 


$$
2 \int d^{2} \mathbf{r} \rho_{0}(\mathbf{r}) \phi_{\mathbf{m}}^{*}(z) \phi_{\mathbf{n}}(z)=\omega_{\mathbf{m}} \delta_{\mathbf{n}, \mathbf{m}}
$$

Next, we multiply relation (17) by $\phi_{\mathbf{m}}\left(z^{\prime}\right)$ and sum over $\mathbf{m}$, apply the completeness relation (16), and finally we obtain the equation for the functions $\phi_{\mathbf{n}}(z)$ :

$$
\frac{1}{2} \omega_{\mathbf{n}} \phi_{\mathbf{n}}(z)=-\partial_{z} \partial_{\bar{z}} \phi_{\mathbf{n}}(z)-\partial_{\bar{z}} \ln \rho_{0}(z) \partial_{z} \phi_{\mathbf{n}}(z)-\left(\partial_{z} \partial_{\bar{z}} \ln \rho_{0}(z)\right) \phi_{\mathbf{n}}(z)-\lambda \pi \rho_{0}(z) \phi_{\mathbf{n}}(z)
$$

Since we are interested in the excitations around a specific ground state, namely, the one given in (17), we use Eq. (41) to rewrite the last two terms in Eq.(18) and look for the solution in terms of a new function $\psi_{\mathbf{n}}(r, \varphi)=\phi_{\mathbf{n}}(r, \varphi) \sqrt{\rho_{0}(r)}$ :

$$
\omega_{\mathbf{n}} \psi_{\mathbf{n}}(r, \varphi)=-\frac{1}{2}(\nabla-i \mathbf{A})^{2} \psi_{\mathbf{n}}(r, \varphi)-\frac{1}{2}\left(v \delta(\mathbf{r})+\lambda N \frac{\delta\left(r-r_{0}\right)}{r_{0}}\right) \psi_{\mathbf{n}}(r, \varphi) .
$$

Equation (19) can be interpreted as a Schrödinger equation for the bosonic particle in the magnetic field and in the additional delta-function potential, where

$$
A_{r}(r, \varphi)=0, A_{\varphi}(r, \varphi)=-\frac{N \lambda}{2 r} \operatorname{sign}\left(r-r_{0}\right), B=\nabla \times \mathbf{A}=-\frac{N \lambda}{r} \delta\left(r-r_{0}\right) .
$$

Notice that we can omit the 'vorticity' term in Eq. (19), because for $r \rightarrow 0$ the wave function must vanish at least as $r^{2}$. The operator on the right-hand side of Eq. (19) commutes with the angular momentum operator $(-i \partial / \partial \varphi)$, so we extract a factor $\exp ( \pm i n \varphi)$ from the eigenfunction, and obtain:

$$
\frac{1}{r} \partial_{r}\left(r \partial_{r}\right) \tilde{\psi}_{0}(r)+\left[2 \omega_{n}-\frac{1}{r^{2}}\left(\frac{N \lambda}{2} \operatorname{sign}\left(r-r_{0}\right) \pm n\right)^{2}\right] \tilde{\psi}_{0}(r)+\frac{N \lambda}{r_{0}} \delta\left(r-r_{0}\right) \tilde{\psi}_{0}(r)=0
$$

where $\tilde{\psi}_{0}(r)$ represents the lowest-energy solution. We have two classes of solution. The first one is

$$
\psi_{n}(r, \varphi)=\exp ( \pm i n \varphi) \times\left\{\begin{array}{l}
J_{\nu}(x) \text { for } r<r_{0} \\
N_{\mu}(x) \text { for } r>r_{0}
\end{array}\right.
$$

and the second one is

$$
\psi_{n}(r, \varphi)=\left\{\begin{array}{l}
\exp ( \pm i n \varphi) J_{\nu}(x) \text { for } r<r_{0} \\
\exp (\mp i n \varphi) N_{\nu}(x) \text { for } r>r_{0}
\end{array}\right.
$$



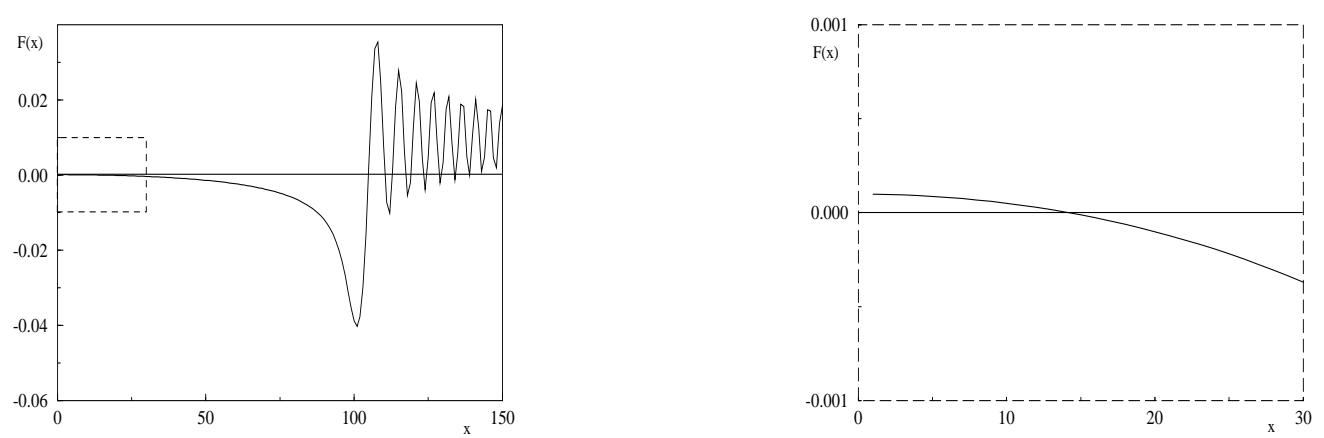

FIG. 1. On the left, the function $F(x)$ is plotted for $\nu=\mu=101, N \lambda / 2=100$. The area in the small box is shown enlarged on the right.

where $J_{\nu}(x)$ and $N_{\nu}(x)$ are Bessel functions, $x=\sqrt{2 \omega_{n}} r$, and $\mu(\nu)=N \lambda / 2 \pm n(N \lambda / 2 \mp n)$. To find out the energy of the solutions (22) and (23), we have to solve the equation of the type

$$
F(x) \equiv J_{\nu}(x) N_{\nu(\mu)}(x)-\frac{2}{N \lambda \pi}=0,
$$

using the expansion for Bessel function for large $\nu(\mu)$ [10]. For small $x$, i. e., for low-energy fluctuations, the function $F(x)$ decreases monotonously and has only one zero, and this is precisely what we are looking for. We illustrate the behavior of the function $\mathrm{F}(\mathrm{x})$ in Fig. 11. Notice that the next zero of the function $F(x)$ is at $x \sim \nu$. This means that $\omega_{n} \gtrsim N^{2} / r_{0}^{2}$, and it costs infinite amount of energy to reach the next state. Effectively, our lowest-energy state is the only relevant state in large $N$. Solving Eq. (24) for $\mu \neq \nu$, we obtain the zero mode $\left(\omega_{n}=0\right)$, but for $\mu=\nu$ we have $2 \omega_{n} r_{0}^{2}=4 n^{2} \mp n N \lambda$. Since $\omega_{n}$ is positive by definition, we finally have

$$
\psi_{n}(r, \varphi)=\left\{\begin{array}{l}
\exp (-i|n| \varphi) J_{\nu}(x) \text { for } r<r_{0} \\
\exp (+i|n| \varphi) N_{\nu}(x) \text { for } r>r_{0}
\end{array}\right.
$$

where $\nu=\frac{1}{2} N \lambda+|n|$ and

$$
\omega_{n}=\frac{1}{r_{0}^{2}}\left(2 n^{2}+|n| \frac{N \lambda}{2}\right)
$$

We can compare the above relation with the dispersion relation of fluctuations around the constant solution in the Sutherland model on a circle of radius $r_{0}$ [11, 12]: 


$$
\omega_{n}^{s}=\frac{1}{r_{0}^{2}}\left(\frac{1-\lambda_{s}}{2} n^{2}+\frac{N_{s} \lambda_{s}}{4}|n|\right) .
$$

In order to establish a full correspondence, we should rescale the dispersion relation (26) as $\omega_{n} \rightarrow 4 \omega_{n} /\left(1-\lambda_{s}\right)$, and demand a sort of statistical transmutation given by

$$
N \lambda=\frac{2 N_{s} \lambda_{s}}{1-\lambda_{s}}
$$

It is interesting to note that the above relation is invariant under the duality transformation $\lambda_{s} \rightarrow 1 / \lambda_{s}$, with $N_{s} \rightarrow-\lambda_{s} N_{s}$, reflecting the well-known symmetry of the Sutherland model [12.

We will now show how our result can be extended to more general vortex solution then that which is concentrated at the origin. The ground-state solution of Eq. (仹) is given in the terms of the analytic function $f(z)$ :

$$
\rho_{0}(z, \bar{z})=\frac{2}{\lambda \pi} \frac{\left|f^{\prime}(z)\right|^{2}}{\left[1+|f(z)|^{2}\right]^{2}}=\frac{1}{2 \lambda \pi}\left|\frac{f^{\prime}}{f}\right| \frac{1}{\cosh ^{2} \ln |f|} .
$$

Let us investigate configurations of vortices positioned at the origin and around it at the locations $z_{i}$ such that outside the circle of radius $R$ there are no vortices $\left(\left|z_{i}\right|<R\right)$. Then $f$ is a polynomial in $z / R$ the degree of which is determined by the normalization condition. At the origin, $f$ goes like $(z / R)^{\alpha N}, \alpha \lesssim 1$. The vortices are positioned at the zeros of $f^{\prime}$. We have a strong vortex at $z=0$, and other vortices are inside the circle. Owing to the normalization condition

$$
\begin{aligned}
N & =\int d x d y \rho(z, \bar{z})=\frac{i}{2 \lambda \pi} \int_{\mathbf{R}^{2}} d \omega_{1}=\frac{i}{2 \lambda \pi} \int_{\partial \mathbf{R}^{2}} \omega_{1}= \\
& =\frac{i}{2 \lambda \pi} \int_{\partial \mathbf{R}^{2}}\left(\frac{f d \bar{f}}{1+f \bar{f}}-\frac{d f \bar{f}}{1+f \bar{f}}\right)
\end{aligned}
$$

$\ln |f|$ is proportional to $N$. Now, $\cosh ^{-2} \ln |f|$ and therefore $\rho_{0}(z, \bar{z})$ is strongly peaked at $|f|=1$, up to $\mathcal{O}\left(1 / N^{2}\right)$, with the width $\Delta \propto 1 / N$. The condition $|f(z / R)|=1$ describes a closed string which in the large- $N$ limit approaches the circle of radius $R$. For illustration, take a simple example

$$
f(z)=\left(\frac{z}{R}\right)^{n}\left(\left(\frac{z}{R}\right)^{m}-1\right)
$$


where $n, m$ are of order $N, n+m=N$. This describes the strong vortex at the origin and a certain number of equidistant vortices positioned on the circle around the origin. $\rho_{0}(z, \bar{z})$ has a maximum on the closed string determined from $|f|=1$ :

$$
\left(\frac{r}{R}\right)^{2(n+m)}-2 \cos (n+m) \varphi\left(\frac{r}{R}\right)^{n+m}+\left(\frac{r}{R}\right)^{2 n}=1 .
$$

In the large- $N$ limit, the equation $r=r(\varphi)$ is approaching a circle of radius $r=R+$ $\mathcal{O}(1 / N)$. Therefore in the large- $N$ limit, we have a bulk-to-edge dimensional reduction: $\lim _{N \rightarrow \infty} \rho_{0}(z, \bar{z}) \rightarrow \delta(|f|-1) \rightarrow \delta(r-R)$.

In conclusion, we can say that in the large- $N$ limit the dynamics of low-lying density excitations around the vortex configuration in the Jackiw-Pi model is equivalent to that of the Sutherland model. Further study is still needed to fully understand the physical meaning of this dimensional reduction and the statistical transmutation associated with it.

\section{Acknowledgment}

This work was supported by the Ministry of Science and Technology of the Republic of Croatia under Contract No. 00980103. 


\section{REFERENCES}

[1] R. Jackiw and S. Templeton, Phys. Rev. D23, 2291 (1981); J. Schonfeld, Nucl. Phys. B185, 157 (1981); S. Deser, R. Jackiw, and S. Templeton, Phys. Rev. Lett. 48, $975(1982)$.

[2] R. Jackiw and S. Y. Pi, Phys. Rev. Lett. 64, 2969 (1990); Phys. Rev. D42, 3500 (1990); Prog. Theor. Phys. Suppl. 107, 1 (1992).

[3] J. Hong, Y. Kim, and P. Y. Pack, Phys. Rev. Lett. 64, 2230 (1990); R. Jackiw and E. Weinberg, Phys. Rev. Lett. 64, 2234 (1990); Z. F. Ezawa, M. Hotta, and A. Iwazaki, Phys. Rev. D44, 452 (1991); P. K. Ghosh, Phys. Rev. D49, 5458 (1994); I. V. Barashenkov and A. O. Harin, Phys. Rev. D52, 2471 (1995).

[4] I. Andrić and V. Bardek, Phys. Rev. D47, 5546 (1993).

[5] F. Calogero, J. Math. Phys. 10, 2191 (1969); 10, 2197 (1969); J. Math. Phys. 12, 419 (1971); B. Sutherland, Phys. Rev. A4, 2019 (1971); Phys. Rev. A5, 1372 (1972); Phys. Rev. Lett. 34, 1083 (1975).

[6] A. P. Polychronakos, Phys. Lett. B264, 362 (1991); T. H. Hansson, J. M. Leinaas, and J. Myrheim, Nucl. Phys. B384, 559 (1992); L. Brink, T. H. Hansson, S. Konstein, and M. A. Vasiliev, Nucl. Phys. B401, 591 (1993); S. Iso, Mod. Phys. Lett. A9, 2123 (1994); H. Azuma and S. Iso, Phys. Lett. B331, 107 (1994); N. Kawakami, Phys. Rev. Lett. 71, 275 (1993); X. G. Wen, Phys. Rev. Lett. 64, 2206 (1990); S. Iso and S. J. Rey, Phys. Lett. B352, 111 (1995); S. Iso, D. Karabali, and B. Sakita, Phys. Lett. B296, 143 (1992); A. Cappeli, C. A. Trugenberger, and G. R. Zemba, Nucl. Phys. B396, 465 (1993); Phys. Rev. Lett. 72, 1902 (1994); J. Avan and A. Jevicki, Phys. Lett. B266, 35 (1991); Phys. Lett. B272, 17 (1991); R. Caracciolo, A. Lerda, and G. R. Zemba, Phys. Lett. B352, 304 (1995).

[7] I. Andrić, V. Bardek, and L. Jonke, hep-th/9507110; Fizika B, (to be published). 
[8] I. Andrić, A. Jevicki, and H. Levine, Nucl. Phys. B215, 307 (1983).

[9] I. Andrić and V. Bardek, J. Phys. A21, 2847 (1988).

[10] I. S. Gradshteyn and I. M. Ryzhik, Table of Integrals, Series and Products (Academic Press, New York, 1980).

[11] I. Andrić, V. Bardek, and L. Jonke, unpublished.

[12] J. A. Minahan and A. P. Polychronacos, Phys. Rev. B50, 4236 (1994). 